\title{
b-Physics at CDF and Prospects for the Next Run
}

\author{
H. Wenzel, INFN-Pisa \\ representing the CDF Collaboration
}

\author{
Fermi National Accelerator Laboratory \\ P.O. Box 500, Batavia, Illinois 60510
}

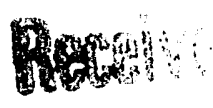

041281991

\section{DISCLAIMER}

\begin{abstract}
This report was prepared as an account of work sponsored by an agency of the United States Government. Neither the United States Government nor any agency thereof, nor any of their employees, makes any warranty, express or implied, or assumes any legal liability or responsibility for the accuracy, completeness, or usefulness of any information, apparatus, product, or process disclosed, or represents that its use would not infringe privately owned rights. Reference herein to any specific commercial product, process, or service by trade name, trademark, manufacturer, or otherwise does not necessarily constitute or imply its endorsement, recommendation, or favoring by the United States Government or any agency thereof. The views and opinions of authors expressed herein do not necessarily state or reflect those of the United States Government or any agency thereof.
\end{abstract}

\section{September 1991}

* Presented at the 3rd Topical Seminar on Heavy Flavours, San Miniato, Italy, June 17-21, 1991. 


\title{
Proceedings of the 3rd Topical Seminar on Heavy Flavours, San Miniato, Italy, 17-21 June 1991 \\ b-Physics at CDF and Prospects for the Next Run
}

\author{
Hans Wenzel, INFN-Pisa \\ for the CDF Collaboration
}

\begin{abstract}
Current CDF b-physics results are presented. The analysis is based on data corresponding to an integrated luminosity of $4.4 p b^{-1}$ recorded with the CDF detector in 88-89 at the Fermilab Tevatron pip collider $(\sqrt{s}=1.8 \mathrm{TeV})$. Preliminary results include the differential cross section $\frac{d \sigma(b)}{d P_{t}}$, some reconstructed exclusive B-decays, a limit for the rare decay $B^{0} \rightarrow \mu^{+} \mu^{-}$and a measurement of $B \bar{B}$ - mixing parameters. Finally we will discuss the prospects concerning b-physics for the next data run which will start in February 1992.
\end{abstract}

\section{Introduction}

The CDF detector for the Fermilab Tevatron $p \bar{p}$ collider $(\sqrt{s}=1.8 \mathrm{TeV})$ combines good tracking $\left(\frac{\delta P_{1}}{P_{1}^{2}} \simeq 0.002\right.$ $\left.(\mathrm{GeV} / \mathrm{c})^{-1}\right)$ with calorimetry which gives it good lepton identification capabilities A detailed description of the detector can be found in reference [1]. The detector and its triggers are optimized for high $P_{t}$ physics to maximize the signal from W's, Z's and top quarks and so it is in principle not well suited to do b physics. Fortunately rature provides us with a very high $b \bar{b}$ production cross section at the Tevatron. So that, although we can access only high $P_{t}$ central b's decaying into a lepton + $\mathrm{x}$ or a $\mathrm{J} / \psi+\mathrm{x}$, it is possible to do a lot of interesting b-physics with the CDF detector.

\section{The b-quark production cross} section $\frac{d \sigma(b)}{d P_{t}}$

The cross section for b-quarks with a $P_{t}>15 \mathrm{GeV}$ was measured [2] using the inclusive electron sample which consists of $0.2 \mathrm{pb}^{-1}$ collected with a $7 \mathrm{GeV}$ trigger threshold and $4.4 \mathrm{pb}^{-1}$ collected with a $12 \mathrm{GeV}$ threshold.

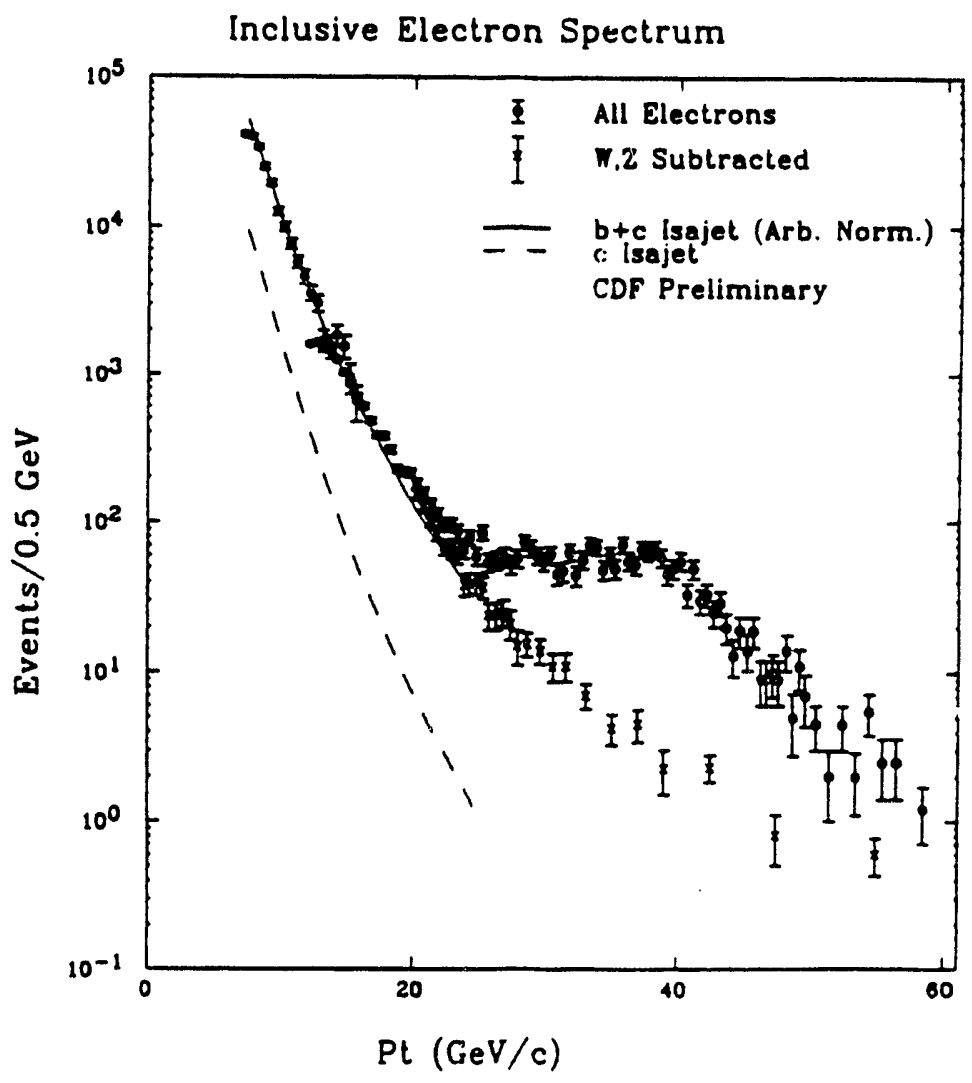

Figure 1: Inclusive Electron Spectrum for $|\eta|<1$.

Figure 1 shows the observed electron $P_{t}$ spectrum for the central pseudorapidity region $|n|<1$., before and after $\mathrm{W}$ and $\mathrm{Z}$ subtraction. The solid line shows the 
prediction of the ISAJET Monte Carlo normalized to ihe experimental data. ISAJET reproduces the $P_{t}$ spec:rum as calculated by Nason, Dawson and Ellis [3]. The dashed line shows the contribution from charm decays as predicted by ISAJET. According to ISAJET $90 \%$ of the electrons in our data sample are from semileptonic b-decays with a $10 \%$ background from charm-decays.

The shape of the distribution agrees very well with the theoretical prediction.

To check if these electrons are really coming from semileptonic b-decay we searched for associated charm within a cone of $\sqrt{\Delta \eta^{2}+\Delta \phi^{2}}<0.6$ around the electron direction. We observe a clear $D^{0} \rightarrow K \pi$ peak (Fig. 2).

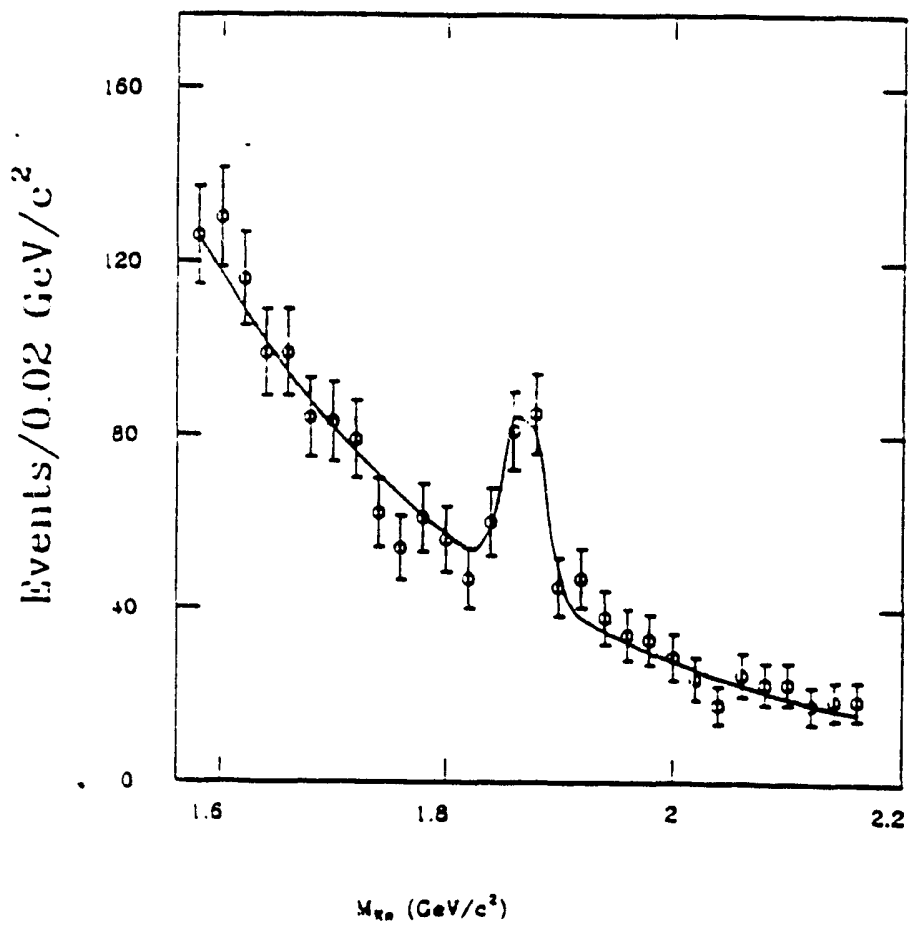

Figure 2: Invariant Mass Spectrum of $K \pi$ pairs for hadron tracks near a high $P_{1}$ electron.

Furthermore we checked the invariant mass spectrum of the e- $D^{0}$-system. The spectrum peaks below the mass of the B-meson as expected because of the missing momentum carried by the neutrino. This proves that we are not dealing with charm pair production close in phase space where one charm particle decays semileptonically
From the electron $P$, spectrum we derived the b-quark cross section as a function of $P_{t}$. In Figure 3 the result is compared with the theoretical prediction [3].

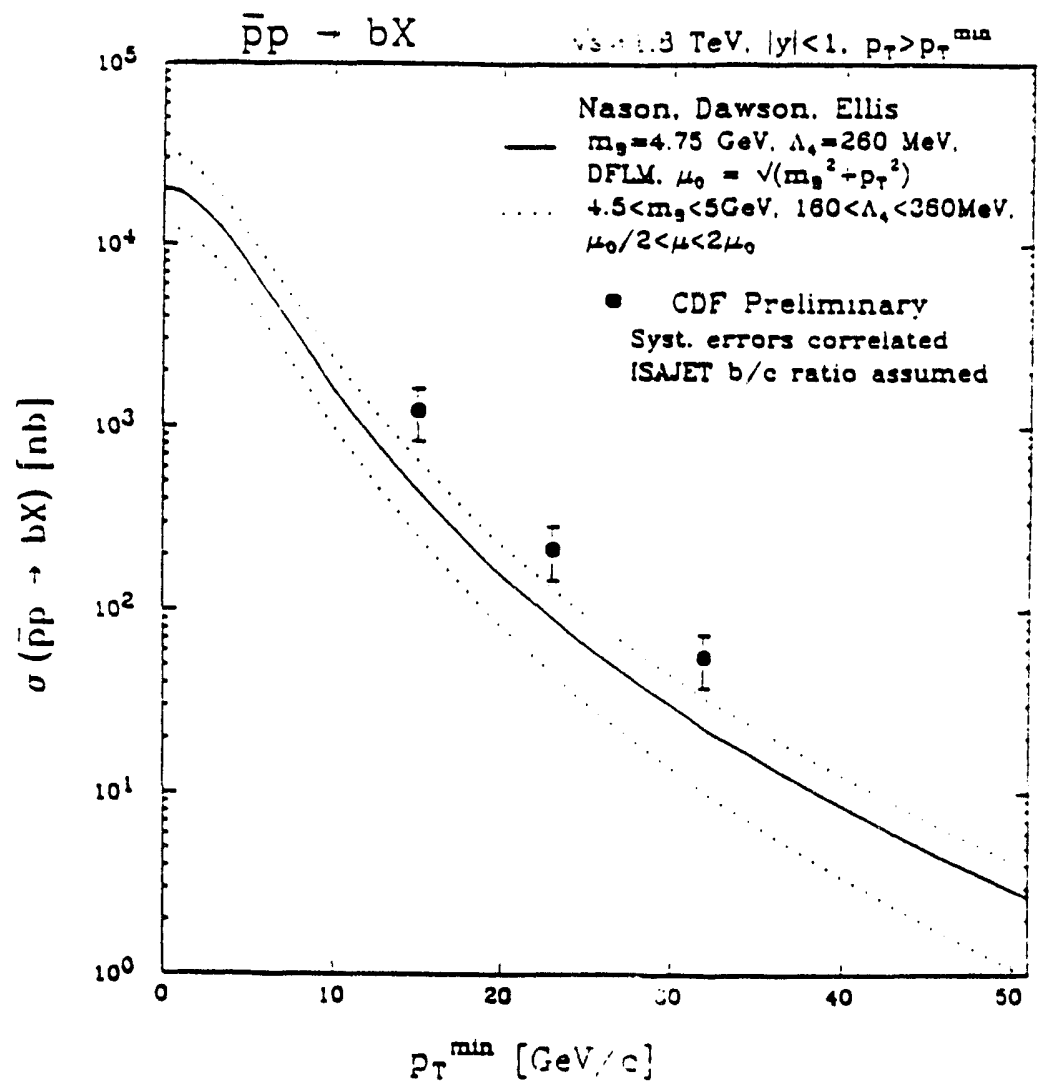

Figure 3: b-quark cross section as a function of b-quark $P_{\text {. }}$.

\section{Reconstruction of exclusive decays.}

To reconstruct exclusive B-decays we used the $\mathrm{J} / \psi \rightarrow$ $\mu^{+} \mu^{-}$data sample [4]. As shown in Figure 4 we observe a very clean $\mathrm{J} / \psi$ peak over a small background. The width of the gaussian distribution fitted to the data is consistent with the excellent momentum resolution of the central driftchamber.

The $J / \psi$ data was maidy collected using the central dimuon trigger. This trigger required two muon candidates with a $P_{\imath}>3 \mathrm{GeV}$ within the acceptance of the 


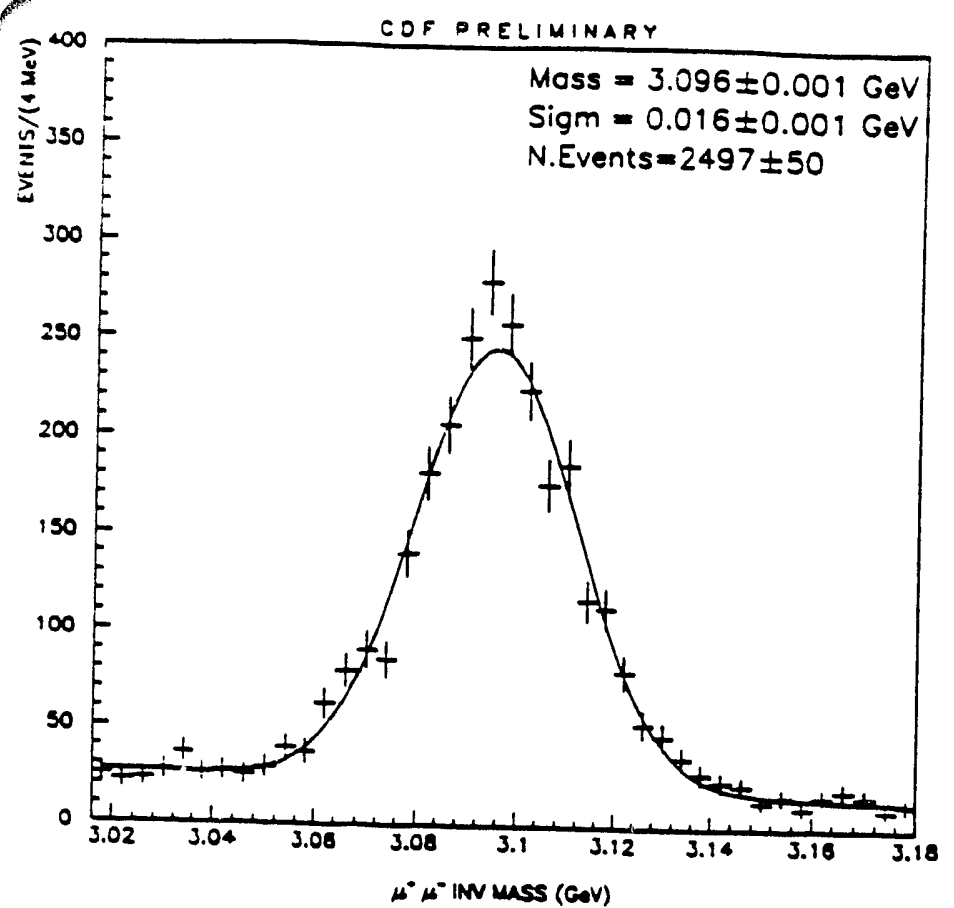

Figure 4: $\mathrm{J} / \psi \rightarrow \mu^{+} \mu^{-}$sample.

cept only very central high $P_{t} J / \psi$ 's. Their mean $P_{t}$ is $8 \mathrm{GeV}$. It is expected [5] that a large fraction of these $\mathrm{J} / \psi$ 's comes from B-decays.

If the $J / \psi$ 's come from B-decay the mean $P_{t}$ of the parent $\mathrm{B}$-meson is about $12 \mathrm{GeV}$ and its decay products are boosted into its direction which is approximately the $\mathrm{J} / \psi$ direction. Firthermore the $\mathrm{J} / \psi$ 's are heavy so that a large fraction of all the decays including a $\mathrm{J} / \psi$ are 2 and 3 body decays.

We reconstructed the two decays: $\mathrm{B}^{ \pm} \rightarrow \mathrm{J} / \psi \mathrm{K}^{ \pm}$and $\mathrm{B}^{0} \rightarrow \mathrm{J} / \psi \mathrm{K}^{0 \bullet} \rightarrow \mathrm{J} / \psi \mathbb{K} \pi$.

The first step of reconstruction is in both cases the same: we find the $\mathrm{J} / \psi \rightarrow \mu^{+} \mu^{-}$and do a mass constrained fit of the $\mathrm{J} / \psi$ tracks to improve the mass resolution for the B-meson. Then for the decay $B^{ \pm} \rightarrow J / \psi K^{ \pm}$ we beam constrain all other tracks assuming that these are kaons and select all tracks with a $P_{6}>2.5 \mathrm{GeV}$ which are within a cone of $60^{\circ}$ around the $J / \psi$. Finally we calculate the invariant mass of the $\mathrm{J} / \psi \mathrm{K}$ pair.

For the $\mathrm{B}^{0} \rightarrow \mathrm{J} / \psi \mathrm{K}^{0} \rightarrow \mathrm{J} / \psi \mathrm{K} \pi$ we select the 3 highest momentum tracks within a cone of $60^{\circ}$ around the
$\mathrm{J} / \psi$, then we form opposite signed track pairs using bot? possible assignments of $K$ and $\pi$ mass and accept only $F$ $\pi$ pairs where the invariant mass is within $50 \mathrm{MeV}$ of the $\mathrm{K}^{0 *}$ mass. Figure 5 shows the invariant mass spectrum. for both decay modes combined. The fit superimposec to the spectrum gives $5279 \mathrm{MeV}$ for the B-mass. The total number of events is $35 \pm 9$.

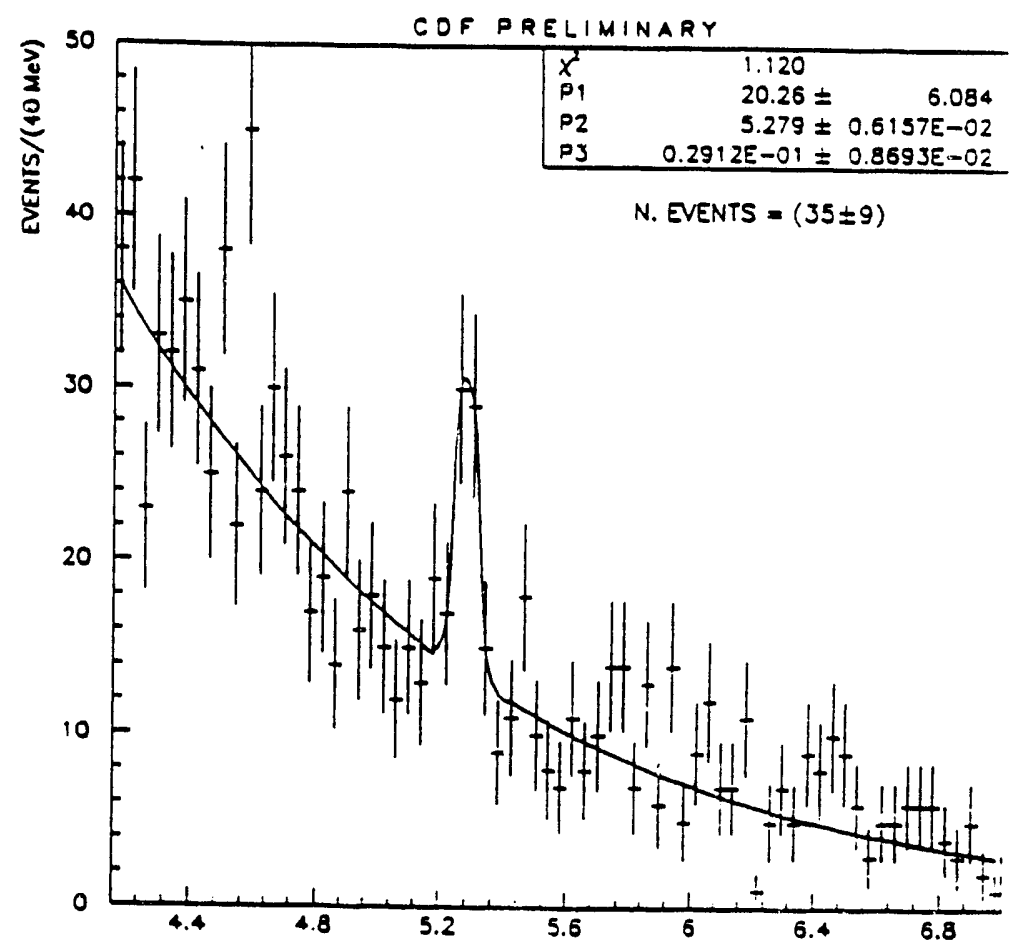

Figure 5: Invariant Mass Specirum $\left(\mathrm{B}^{0} \rightarrow \mathrm{J} / \psi \mathrm{K}^{* 0}\right.$ and $\mathrm{B}^{ \pm} \rightarrow \mathrm{J} / \psi \mathrm{K}^{ \pm}$combined).

\section{A limit for the rare decay $\mathrm{B}^{0} \rightarrow \mu^{+} \mu^{-}$}

We searched for the rare decay $B^{0} \rightarrow \mu^{+} \mu^{-}[6]$, which is a flavor changing neutral current decay allowed by the standard model at very low rate. The expected branching ratio is [7]: $\mathrm{BR}\left(\mathrm{B}^{0} \rightarrow \mu^{+} \mu^{-}\right) \simeq 10^{-8}$ to $10^{-9}$.

To derive a limit for the branching ratio, we compare this decay with the decay $B \rightarrow \psi^{\prime} X \rightarrow \mu^{+} \mu^{-X}$. We assume that all the $\psi$ "s are from B decay and that $50 \%$ of them are from neutral and $50 \%$ are from charged $B$ 
mesozs. The branching ratio $\mathrm{BR}\left(\mathrm{B} \rightarrow \psi^{\prime} \mathrm{x} \rightarrow \mu^{+} \mu^{-} \mathrm{X}\right)$ is $2.5 \pm 1.2 \cdot 10^{-5}$. By measuring the rate ratio of the two decays we find that systematic uncertainties in trigger efficiency, b-production properties, luminosity etc., cancel. The acceptance ratio $\epsilon_{\text {ace }}$ was calculated using Monte Carlo's to be:

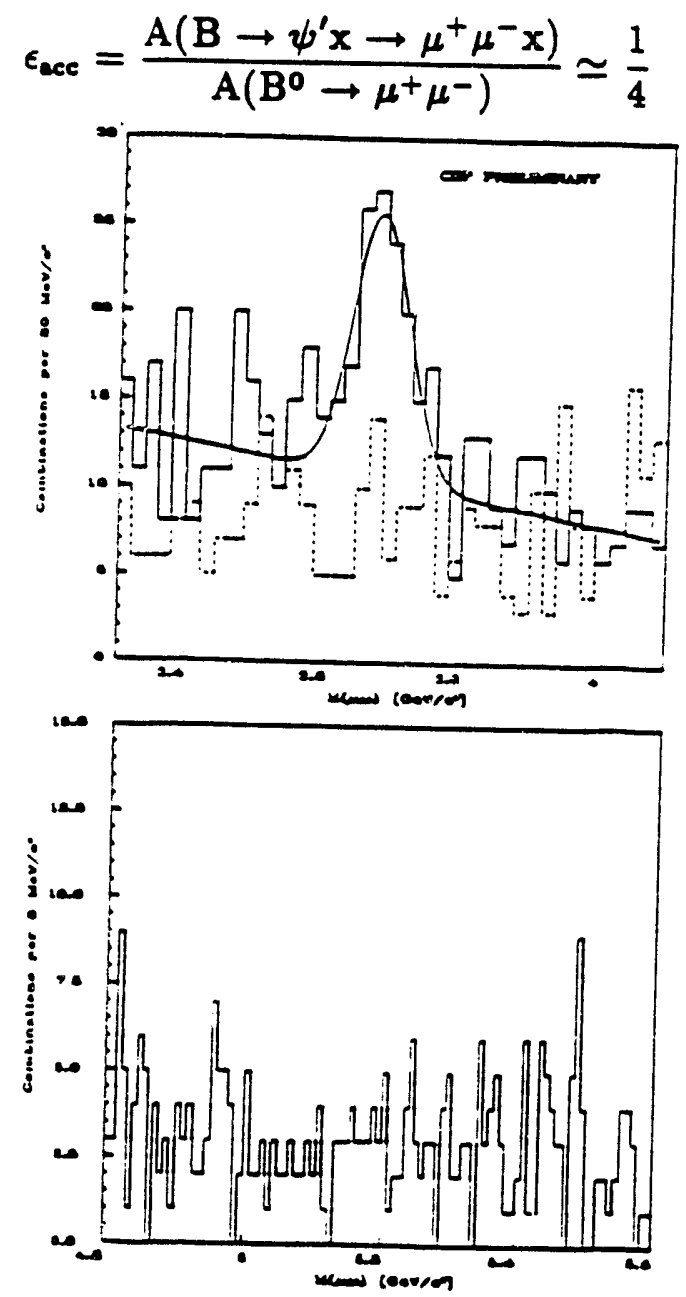

Figure 6: $\mu^{+} \mu^{-}$-Invariant Mass Spectrum in the a.) $\psi^{\prime}$ and $b$.$) in the B$-meson mass region.

Figure 6 b.) demonstrates that the invariant mass distribution in the $B^{0}$-mass region is consistent with a flat background. The fit gives an upper limit of 12 events at $90 \%$ confidence level compared with $72 \pm 17$ events in the $\psi^{\prime}$-mass region. So we obtain the following ratio of the rates:

$$
\frac{N\left(B^{0} \rightarrow \mu^{+} \mu^{-}\right)}{\mathrm{N}\left(\mathrm{B} \rightarrow \psi^{\prime} \mathrm{x} \rightarrow \mu^{+} \mu^{-} \mathrm{x}\right) \cdot 0.5} \cdot \epsilon_{\text {ace }}=
$$

$$
\frac{12}{(72 \pm 17) \cdot 0.5} \cdot 0.25
$$

This gives: $\mathrm{BR}_{(}\left(\mathrm{B}^{0} \rightarrow \mu^{+} \mu^{-}\right)<3.2 \cdot 10^{-6}$ at $90 \%$ Confidence Level. This is the most stringent existing limit. The UA1-collaboration quotes: $\mathrm{BR}\left(\mathrm{B}^{0} \rightarrow \mu^{+} \mu^{-}\right)<8.3$. $10^{-6}$ in [8]. In the Particle Data Book [9] we find $\mathrm{BR}\left(\mathrm{B}^{0} \rightarrow \mu^{+} \mu^{-}<5 \cdot 10^{-5}\right)$.

\section{Measurement of $\mathrm{B}^{0} \overline{\mathrm{B}}^{0}$ Mixing using e $\mu$-events}

$\mathrm{B}^{0} \overline{\mathrm{B}}^{0}$ mixing is a second order weak interaction effect which originates from the box diagrams shown in Fig. 7. It depends on the B-meson lifetime, the elements of the CKM matrix and the masses of the $b$ and top quarks. So measuring mixing parameters gives access to parameters of the Standard Model and allows to check its validity. Mixing has been studied at $e^{+} e^{-}$-machines and at the Spps Collider [10].

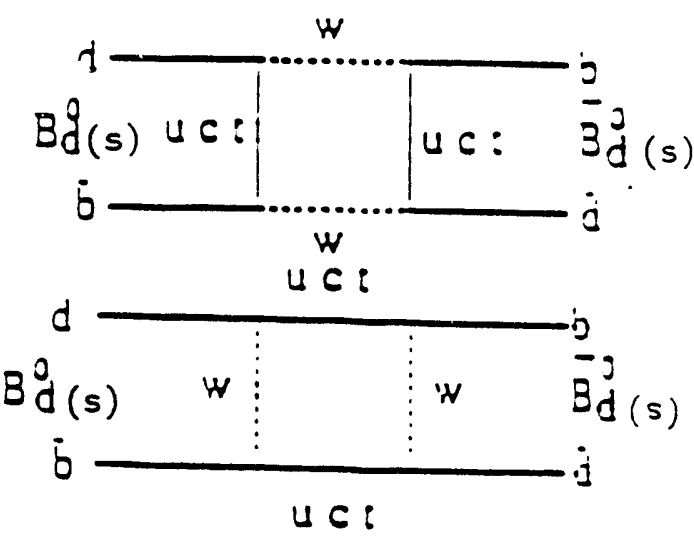

Figure 7: Box diagrams for $\mathrm{B}^{\circ} \overline{\mathrm{B}}^{0}$ Mixing.

When using events where both b's in a b $\bar{b}$-event decay semileptonically the charge of the leptons tags the flavor of the b-quark. Mixing is a source of like sign (LS) lepton pairs. The mixing parameter $\chi$ gives the probability of $B^{0} \bar{B}^{0}$ mixing in $b \bar{b}$-events, where $B^{0}$ refers to $B_{d}^{0}$ or $B_{y}^{0}$. So this parameter is the average of the $B_{d}^{0}$ and $B_{s}^{0}$ mixing 
parameters $\chi_{\mathrm{d}}$ and $\chi_{\mathbf{s}}$

$$
\bar{\chi}=P_{\mathrm{d}} \chi_{\mathrm{d}}+P_{\mathbf{s}} \chi_{\mathbf{s}}
$$

where

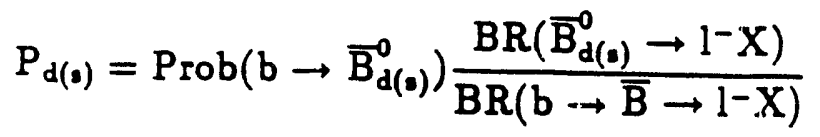

$\bar{\chi}$ is related to the ratio $\mathrm{R}$ between LS and opposite sign (OS) lepton pairs in the following way:

$$
R=\frac{N(\text { LS })}{N(\text { OS })}=\frac{2 \bar{\chi}(1-\bar{\chi})+\left[(1-\bar{\chi})^{2}+\bar{\chi}^{3}\right] f_{s}}{\left[(1-\bar{\chi})^{2}+\bar{\chi}^{2}\right]+2 \bar{\chi}(1-\bar{\chi}) f_{s}+f_{c}}
$$

where

f, : Probability of sequential decay $b \rightarrow c X \rightarrow 1 X$

$f_{c}$ : Probability of OS dileptons from $c \bar{c}$

Using e $\mu$-events has the advantage that the rate is high (twice: ee, $\mu \mu$ ) and that there is no background from $\mathrm{DY}, \mathrm{J} / \psi, \mathrm{Y}$.

After applying CDF standard cuts for electrons and

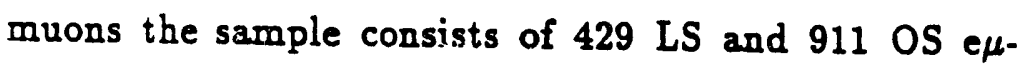
events [11]. The $P_{t}$ threshold is $5 \mathrm{GeV}$ for the electrons and $3 \mathrm{GeV}$ for the muons. Figure 8 shows the invariant mass distribution for LS and OS lepton pairs.

To remove sequential $b$ decays $(b \rightarrow c l X \rightarrow 11 X)$ we

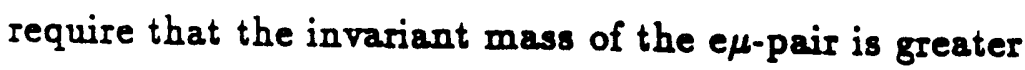
than $5 \mathrm{GeV}$. This cut also preferentially removes pairs with a small relative angle. After this cut we have 346 LS and 554 OS events.

The fake leptons background comes mainly from fake muons. The rate of fake muons has been estimated using other CDF data samples. They contribute equally to IS and OS pairs. So we get the following value for $R$ :

$$
\mathrm{R}=\frac{346-90}{554-90}=0.552 \pm 0.049(\mathrm{stat}) \pm_{0.048}^{0.039}(\mathrm{bkg}) \text {. }
$$

The ISAJET Monte Carlo gives:

$f_{c}=0.066 \pm 0.066$ and $f_{s}=0.248 \pm 0.055$.

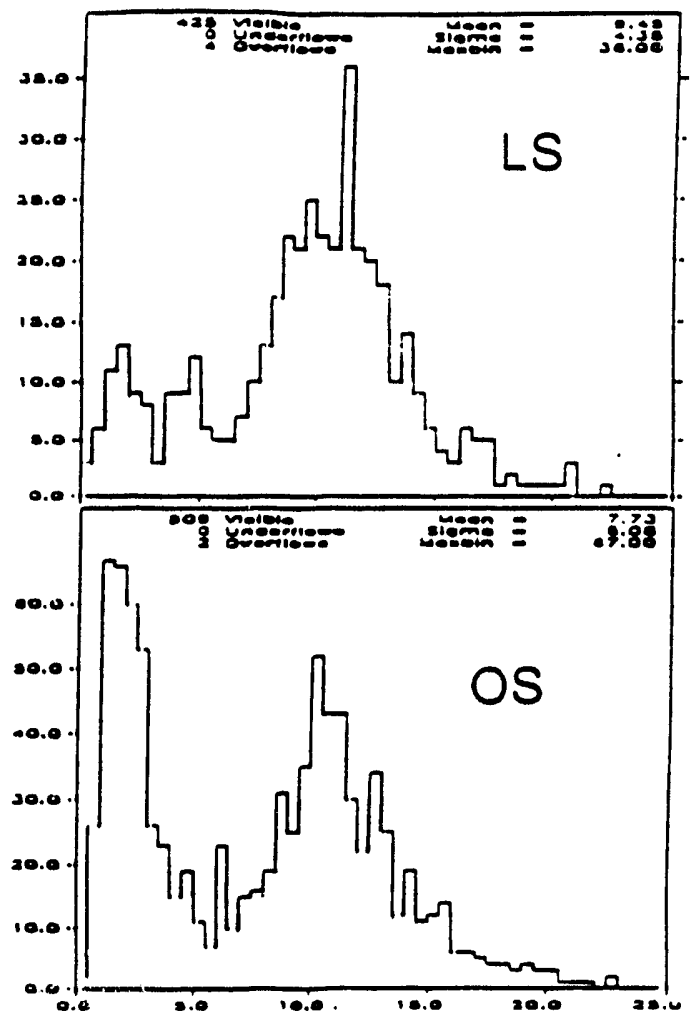

Figure 8: Invariant Mass Distribution for LS and $O S$ lepton pairs.

Finally we obtain the following value for $\bar{\chi}$ :

$\bar{\chi}=0.176 \pm 0.028$ (stat) $\pm 0.025(\mathrm{bkg}) \pm 0.032(\mathrm{MC})$

Within the errors this is in good agreement with the UAl results: $\bar{\chi}=0.148 \pm 0.029$ (stat) \pm 0.017 (sys)

\section{Prospects for the next run}

Analyzing the 88/89 data CDF demonstrated its ability to do b-physics at a hadron collider. The next data run which consist of two parts is expected to start February 92. The first part of the run will last about 6 months and the goal is to record $25 \cdot 30 \mathrm{pb}^{-1}$ on tape during that time. This is about 6 times the luminosity integrated in $88 / 89$. For the second part the goal is more than $50 \mathrm{pb}^{-1}$.

The upgraded CDF-detector will be equipped with a microstrip Silicon Vertex Detector (SVX) (for a description see reference (12]). This device will have an impact 
parameter resolution of about $10 \mu \mathrm{m}$ for tracks with a transverse momentum of more than $1 \mathrm{GeV}$.

In addition, the central CDF muon system will be improved. The muon acceptance which is used in the trigger will be increased by roughly a factor of two and the shielding in the central part $(|\eta|<0.7)$ will be increased so that the background from hadron punch through will be suppressed by a factor of five. Both improvements will allow us to lower the trigger thresholds: e.g. in the $88 / 89$ run the central dimuon trigger required both muon candidates to have a $P_{t}$ of $3 \mathrm{GeV}$, in the next run this threshold will be $1.8 \mathrm{GeV}$. For the dimuons we are expecting an overall increase of trigger efficiency by a factor of 16 . We expect to fully reconstruct more than $3000 \mathrm{~B}$ 's in the $\mathrm{B} \rightarrow \mathrm{J} / \psi+\mathrm{X}$ channel in $25 \mathrm{pb}^{-1}$.

This data sample will be a good starting point to do bspectroscopy and we hope to discover the not yet found lugher mass states of the b-quark in the following channels: $\mathrm{B}, \rightarrow \mathrm{J} / \psi \varphi \rightarrow \mathrm{J} / \psi \mathrm{KK}, \Lambda_{\mathrm{b}} \rightarrow \mathrm{J} / \psi \Lambda \rightarrow \mathrm{J} / \psi \mathrm{p} \pi$ $\mathrm{B}_{\mathrm{c}} \rightarrow \mathrm{J} / \psi \pi \ldots$

With the SVX it will be possible to reduce the combinatorial background significantly by assigning the B-decay candidate tracks to a common vertex and to measure the lifetimes of the different $B$ hadrons $\left(B^{0}, B^{ \pm}, B_{2}, \Lambda_{b}\right)$ separately. Of particular interest will be the reconstruction of the decay $\mathrm{B}^{0} \rightarrow \mathrm{J} / \psi \mathrm{K}_{\mathrm{S}}^{0}$ where the final state is a $C P$-eigenstate and a promising candidate for the future measurement of $C P$-violation in the $B \bar{B}$ system.

Besides improving our limit for the decay $\mathrm{B}^{0} \rightarrow \mu^{+} \mu^{-}$ we will search for the so called 'Penguin'-decay $b \rightarrow 8 \mu \mu$.

Also the single muon and single electron triggers will be improved in the next rur. We expect 30000 leptons from b-decay per $\mathrm{pb}^{-1}$ for each of these triggers. Depending on the performance of the SVX it will he mos- sible to make an unbiased study of the second $b$ in the event.

\section{References}

[1] F. Abe et al., CDF collaboration, The CDF Detector: An Overview ; NIM A271 (1988), page 387-403

[2] J. Proudfoot, F. Ukegawa and A. B. Wicklund Measuring the Bottom Quark Cross Section Using the Inclusive Electrons ; CDF internal note 1253 (1990)

[3] P. Nason, S. Dawson, R.K. Ellis

The One Particle Inclusive Differential Cross Section for Heavy Quark Production in Hadronic Collisions ; Nuclear Physics B327 (1989), page 49-92

[4] A. Sansoni, V. Scarpine,

Reconstruction of the $B$-meson decays at $C D F$ : CDF internal note 1244 (1990)

[5] Glover, Martin, Stirling

Z. Physik 38C (1988),page 473

[6] T. F. Rohaly

$A$ limit on $\mathrm{B}^{0} \rightarrow \mu^{+} \mu^{-}$; CDF internal note 1232 (1990)

[7] Cronin et al.

Proceedings of the 1984 Snowmass Summer Study on the Design and Utilization of the SSC; page 161-169

[8] F. Albajar et al., UA1 collaboration, $A$ Search for Rare B-Meson Decays at the CERN SppS Collider; submitted to Phys. Lett. B, CERNPPE/91-54

[9] Particle Data Group

Phys. Lett. B239(1990)

[10] F. Albajar et al., UA1 collaboration, Measurement of $\mathrm{B}^{0} \overline{\mathrm{B}}^{0}$ Mixing at the CERN Sp $\overline{\mathrm{p}} \mathrm{S}$ Collider ; submitted to Phys. Lett. B, CERNPPE/91-55

[11] R. Hollebeek, L.Song, G.P. Yeh, J. Yoh, Measurement of $\mathrm{B}^{0} \overline{\mathrm{B}}^{0}$ Mixing using Electron-Muor Events ; CDF internal note 1355 (1991)

[12] D. Amidei et al:;

The CDF SVX: A Silicon Vertex Detector for , Hadron Collider ; NIM A289 (1990), page 388-399 

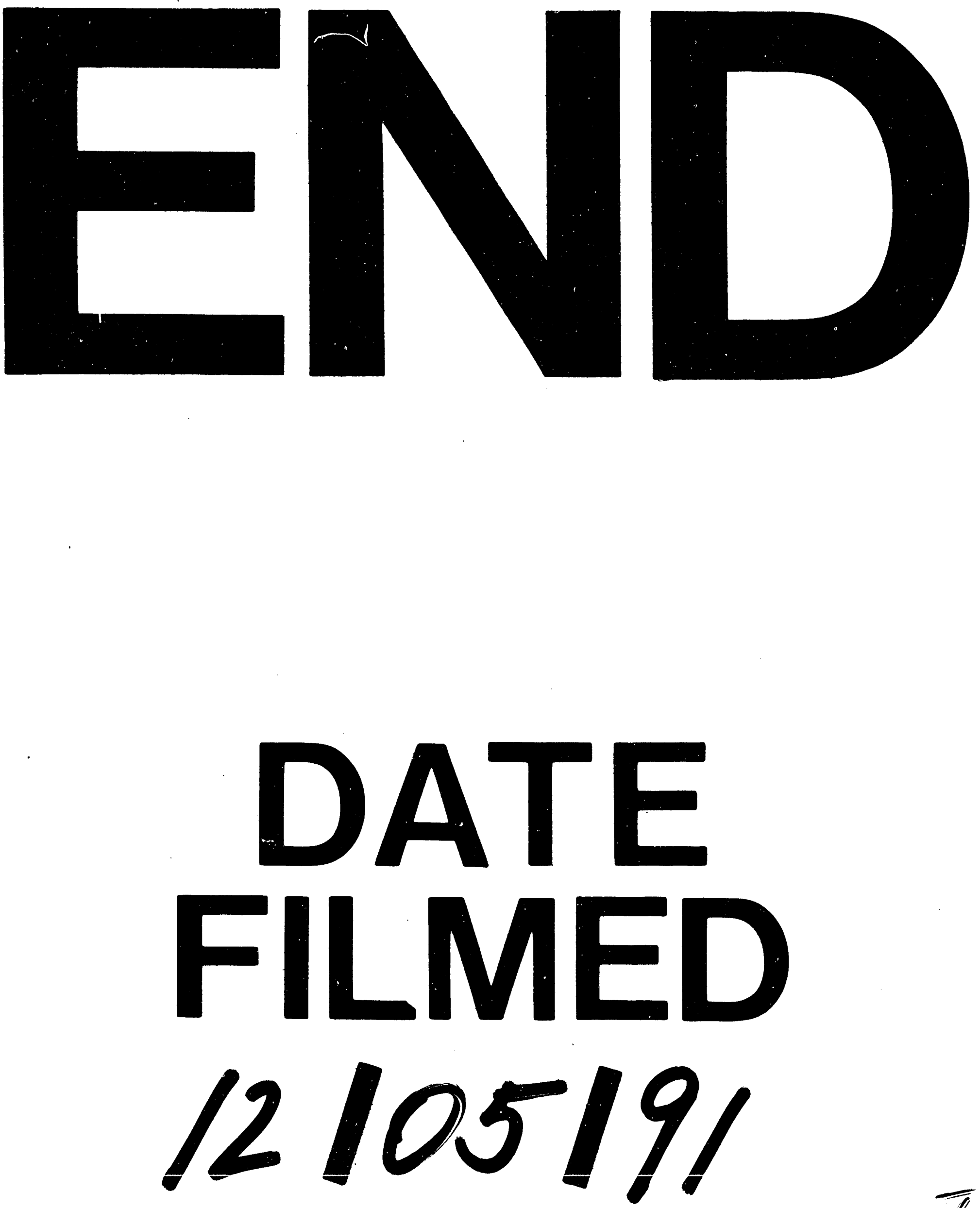
